\title{
Review on Digital Radiography: Open EMR workflow and Wi-Fi Networking System
}

\author{
Hinal Fifadra \\ student, \\ Department of \\ Biomedical Engg., \\ D J Sanghvi College of Engg., \\ Mumbai,India
}

\author{
Deval Parikh \\ student, \\ Department of Biomedical Engg., \\ D J Sanghvi College of Engg., \\ Mumbai,India
}

\begin{abstract}
Few years back X-Ray film based technology was very popular in use. Though it was widely used but it required a lot of improvisation, and digitalization was a very good way. Through Digital Radiography a complete hard copy process got shifted to a complete soft copy one. The advantages of Digital Radiography over the conventional method are mentioned in the paper. There is also a workflow described in this paper. We have also mentioned the advantages and disadvantages of this method (OpenEMR). Methods to detect the problems in the workflow and a wireless system are suggested for preventing the errors caused during the transmission of data. Even this method has flaws but we have suggested few solutions for them.
\end{abstract}

\section{Keywords}

OpenEMR; Digital Radiography; PACS; DICOM; wi-fi network

\section{INTRODUCTION}

Some 20 years ago the X-ray Radiographic field got shifted from physical X-ray films to the digital X-ray images[1]. This change was inexorable because digitalizing had many advantages over the conventional X-ray film techniques. The traditional methods were less reliable, very difficult to use, more exposure to X-rays was observed, it consumed a lot of time, required labor power, expensive and major disadvantage was the printed images existed in single copies[1], so if the Xray image show data losses the complete procedure with the patients had to be repeated. And also according to the FDA's law to keep the patients record for a particular span of time wasn't feasible using the traditional technique [2].Along with the manipulation, quality improvisation and immediate image viewing which was not possible in the older technique is made plausible with Digital Radiography (DR)

Now, Digital Radiography (DR) makes use of digital X-ray sensors like flat panel detectors and high density line scan detectors. For this digitalization Picture Archiving And Communication System (PACS), Radiology Information System(RIS), Electronic Health Care Records(EHR) systems and more complete standards like Digital Imaging And Communications in Medicine(DICOM), Health Layer 7(HL7), Integrating the Health care Enterprise(IHE),are used to provide more holistic patient data capture and disinterested sharing[3]. So proper treatment of Medicare/Medicaid patients
Meaningful Use (MU) declared Stage 1 requirements in 2013 and Stage 2 in 2014.

\section{WORKFLOW OF AN OPENEMR}

In recent Digital Radiography (DR), PACS/RIS containing DICOM files are interfaced with EHRs. One such work flow with OpenEMR is described in this paper using the case study mentioned in a paper [3]. OpenEMR is a software for keeping medical record and it is used for electronic practice management. This workflow is used by $1,00,000$ practitioners worldwide on over 200 million patients.

The workflow is:

- As the patients reach the reception of the hospital their data is looked at.

- The person in charge take the patients for primary examination to enter the chief complains and hence the medical history is upgraded.

- Secondary examination is done by the physician who will decide whether diagnostic tests are required or not. If the tests are necessary then the physician enter them in OpenEMR.

- According to the prescribed test by the physician, inhouse tests are carried out and the results are put on the OpenEMR either through scanning or they are manually transcribed.

- The imaging modality equipment is connected to the PACS on the dedicated computer which exports DICOM images to a DICOM server or an external media. The physician views those images using DICOM viewer.

- Now, the physician can easily carry out diagnosis or if he feels he can advise other tests for clear results. As the diagnosis is done it is recorded on the OpenEMR by the physicians. In OpenEMR, entries of diagnosis is done using International Statistical Classification of Diseases (ICD9) and billing data is entered using Health Care Common Procedure Coding System(HCPCS)/ Current Procedural Terminology(CPT4).

- Scheduling the follow ups and payment collection is done at the reception.

Complete workflow can be easily understood from the flowchart given below in Fig.1 




Fig.1 Work Flow of an OpenEMR system

OpenEMR has many advantages which makes it use wide. Since it can be used on various platforms like Windows, Mac and Linux, making it user friendly. It becomes very helpful due to its easy choosing between single or multiple office installation. All the data can be easily accessed in a secured data connection. And many secured network connections are available. Viewing of images can be done using RadScraper, Mirth Connect web viewers. Other method that can be utilized for image viewing is converting DICOM file to Potable Network Graphics(PNG) or Joint Photographic Experts Group (JPEG).DICOM can be read to CD/DVD. Storage facilities are also available using OpenEMR. And due to its smooth working over a third of users are based in United States [3].

\section{DISADVANTAGES OF OPENEMR}

There are many good things about Digital Radiography. But digitalization has its own cons. Many a times it happens that there are losses in the system, but we don't even realize that there are any. These losses might be negligible sometimes but for diagnostic purposes these losses may hamper the treatment of the patients. Detecting these losses is a task.

How do these losses occur? It happens that some file formats are not compatible with OpenEMR for example DICOM, then a zip file is uploaded [4]. Since there is no direct interface between OpenEMR and DICOM, certain webviewers which are not yet approved by FDA are used [3]. But this is not legal, so people convert the DICOM file to PNG or JPEG which causes compression and hence loss of data. Additionally, encryption while transmitting data are security has to be taken into account. Not only encryption but also deciphering at the receivers end is important. This may cause vendor incompatibility, even if the device is fully DICOM compliant [1]. There might be a complete series loss of images, it might also happen that at the receivers end a series gets added and the most dangerous partial loss one or few images from a series [1]. Partial loss is dangerous because it is very difficult to be traced.

\subsection{Methods to Detect These Losses}

1. One of the methods is visiting a site and personally monitoring it. Monitoring would involve tallying each and every transmitted and received file. Even one less received image can be identified. But this is a very tedious task. It requires a lot of time as well as man power which adds up to the cost.

2. The above shortcomings can be overcome by connecting scanners to remotely via DICOM Query [1]. This made the comparison of sent and received data very easy. The physical presence at the scanner was not required.

But comparing things would always be time consuming so for smooth workflow the devices at the hospital have to be checked after every six months. 


\section{PREVENTIVE MEASURES FOR TRANSMITTING DATA}

The above methods were for detection of the data loss but we have to prevent them from happening during the transmission of the data, since majorly hospitals use PACS, EMRs. For preventing something we need to know the cause of its occurrence. Few causes are mentioned above, there is one more which hinders the data transmission that is connection of Ethernet cables to the Local Accessible Network (LAN) Port for each transmission. This is not feasible always, hence substituting the connection of cables to the port by creating a Wi-Fi network can be used [4].For this, installation of Ethernet to wireless converter is required. There is a rosette types connection where the Access Point (AP) was at the center and it was connected to the LAN Port. The system can be implemented for multiple rooms. With this system if there is any problem at the time of transmission it indicates a message- "Network Error".

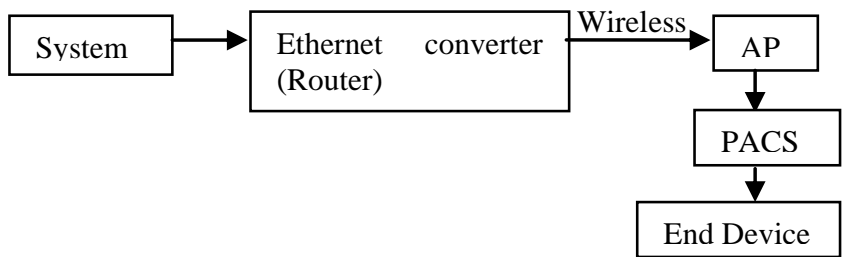

Fig.2 Block Diagram of a wireless transmitting system.

The workflow can be easily understood by the block diagram give above in Fig.2.Though this system is very easy to use but there are shortcomings such as:

- Electromagnetic waves radiated by other devices cause hindrances in transmission of data through Wi-Fi network [4].

- The connection of router for creating Wi-Fi network reduces the speed for data transmissions.

- $\quad$ Bad weather affects the speed of Wi-Fi networks due to lack of proper signals (frequency) [5].

- Power consumption is fairly high compared to some other standards making the battery life shorter and heat is a concern to some users [6].

- Since the workstation unit's Operating System is DOS 6.0 finding compatible wireless Ethernet was also an issue [4].

- $\quad$ Range of the Wi-Fi network created by the router would be limited.

\section{CONCLUSION AND DISCUSSION}

Solutions for some of the above problems:

- Since, Electromagnetic interferences by the other devices is the major problem faced in this method we can use shielding of these devices. This will prevent the Electromagnetic radiation from causing problem in the Wi-Fi network during transmission.

- Range is also an issue to be taken care of, we can use Wi-Fi boosters for broadening the range.

The review is of the conventional X-ray film based method and why it was taken over by DR. The review also consists of a method based on DR., where manipulation of DICOM files was possible by PACS. These files had to be uploaded on OpenEMR according to the suggested workflow. Though this method was very advantageous in DR, it had a major drawback like loss of image data. For detecting these losses two methods are mentioned.

Prevention of problems while transmission is also possible through wi-fi network. It is a very easy solution but again there are few disadvantages for which we have mentioned some solutions. There is a lot of further scope in this topic.

\section{ACKNOWLEDGEMENTS}

We would like to thank the Biomedical department of D J Sanghvi college of engineering for giving us a chance to write this paper and our whole faculty, especially Prof. Purva Badhe for not only including this in our course but also for guiding us throughout the paper. We would also like to thank our seniors who helped us for writing this paper.

\section{REFERENCES}

[1] Oglevee C.,Pianynkh O., "Losing Images in Digital Radiology:More than You Think," J Digit Imaging (2015) 28:264-271

\section{DOI 10.1007/s10278-014-9748-2}

[2] Website:healthandwelfare.idaho.gov/Portals/0/MedicalLi censing Certification/RecordretentionReqs.pdf(Accessed on $2^{\text {nd }}$ March 2016, at 5:00pm).

[3] Piliouras T.,Suss R.,Yu P.,'Digital Imaging \& Electronic Health Record Systems," Systems,Applications and Technology Conference(LISAT),2015 IEEE,Long Island,1-6,Date of conference:1-1 May 2015,DOI 10.1109/LISAT.2015.7160179.

[4] Park H.,Joo S.,Huh S.,'Improvement in the Image Transmission of the Mobile Digital C-Arm X-Ray System in the Operatin Room," 2012 5th International Conference on BioMedical Engineering and Informatics (BMEI 2012).

[5] Website:frndzzz.com/internet-wireless-advantages-anddisadvantages-wifi-internet.(Accessed on $6^{\text {th }}$ March 2016, at 3:00pm).

[6] Website:bwif.org/wifi_disadantages.html.(Accessed on $6^{\text {th }}$ March 2016 at4:00pm). 